\title{
Temsirolimus combined with cyclophosphamide and etoposide for pediatric patients with relapsed/refractory acute lymphoblastic leukemia: a Therapeutic Advances in Childhood Leukemia Consortium trial (TACL 2014-001)
}

Sarah K. Tasian, ${ }^{1,2}$ Lewis B. Silverman, ${ }^{3}$ James A. Whitlock, ${ }^{4}$ Richard Sposto, ${ }^{5}$ Joseph P. Loftus, ${ }^{1}$ Eric S. Schafer, ${ }^{6}$ Kirk R. Schultz, ${ }^{7}$ Raymond J. Hutchinson, ${ }^{8}$ Paul S. Gaynon, ${ }^{9}$ Etan Orgel, ${ }^{9}$ Caroline M. Bateman, ${ }^{10}$ Todd M. Cooper, ${ }^{11}$ Theodore W. Laetsch, ${ }^{1,2}$ Maria Luisa Sulis, ${ }^{12}$ Yueh-Yun Chi, ${ }^{9}$ Jemily Malvar, ${ }^{9}$ Alan S. Wayne ${ }^{9}$ and Susan R. Rheingold ${ }^{1,2}$

\footnotetext{
'Division of Oncology and Center for Childhood Cancer Research, Children's Hospital of Philadelphia, Philadelphia, PA, USA; ${ }^{2}$ Perelman School of Medicine at the University of Pennsylvania, Philadelphia, PA, USA; ${ }^{3}$ Department of Pediatric Oncology, Dana-Farber Cancer Institute and Division of Pediatric Hematology-Oncology, Boston Children's Hospital, Boston, MA, USA; ${ }^{4}$ Division of Haematology/Oncology, Hospital for Sick Children and the University of Toronto, Toronto, Ontario, Canada; ${ }^{5}$ Department of Preventive Medicine, Keck School of Medicine, University of Southern California, Los Angeles, CA, USA; ${ }^{6}$ Dan L. Duncan Institute for Clinical and Translational Research, Baylor College of Medicine and Texas Children's Cancer Center, Houston, TX, USA; ${ }^{7}$ Division of Hematology/Oncology/Bone Marrow Transplant, British Columbia Children's Hospital, Vancouver, British Columbia, Canada; ${ }^{8}$ Division of Hematology/Oncology, CS Mott Children's Hospital, Ann Arbor, MI, USA; 'DDivision of Hematology/Oncology, Children's Hospital Los Angeles, Norris Comprehensive Cancer Center, Keck School of Medicine, University of Southern California, Los Angeles, CA, USA; ${ }^{10} \mathrm{Cancer}$ Centre for Children, The Children's Hospital at Westmead, Westmead, New South Wales, Australia; ${ }^{11}$ Division of Hematology/Oncology, Seattle Children's Hospital Cancer and Blood Disorders Center, Seattle, WA, USA and ${ }^{12}$ Department of Pediatrics, Memorial Sloan Kettering Cancer Center, New York, NY, USA
}

Correspondence: S. R. Rheingold rheingold@chop.edu

$\begin{array}{ll}\text { Received: } & \text { June 25, } 2021 . \\ \text { Accepted: } & \text { December 7, } 2021 . \\ \text { Prepublished: } & \text { February 3, } 2022 .\end{array}$

https://doi.org/10.3324/haematol.2021.279520

๑2022 Ferrata Storti Foundation Published under a CC BY-NC license @ @ (1)

\begin{abstract}
Phosphatidylinositol 3-kinase (PI3K)/mammalian target of rapamycin (mTOR) signaling is commonly dysregulated in acute lymphoblastic leukemia (ALL). The TACL2014-001 phase I trial of the mTOR inhibitor temsirolimus in combination with cyclophosphamide and etoposide was performed in children and adolescents with relapsed/refractory ALL. Temsirolimus was administered intravenously (IV) on days 1 and 8 with cyclophosphamide $440 \mathrm{mg} / \mathrm{m}^{2}$ and etoposide $100 \mathrm{mg} / \mathrm{m}^{2}$ IV daily on days $1-5$. The starting dose of temsirolimus was $7.5 \mathrm{mg} / \mathrm{m}^{2}$ (DL1) with escalation to $10 \mathrm{mg} / \mathrm{m}^{2}$ (DL2), $15 \mathrm{mg} / \mathrm{m}^{2}$ (DL3), and $25 \mathrm{mg} / \mathrm{m}^{2}$ (DL4). PI3K/mTOR pathway inhibition was measured by phosphoflow cytometry analysis of peripheral blood specimens from treated patients. Sixteen heavily-pretreated patients were enrolled with 15 evaluable for toxicity. One dose-limiting toxicity of grade 4 pleural and pericardial effusions occurred in a patient treated at DL3. Additional dose-limiting toxicities were not seen in the DL3 expansion or DL4 cohort. Grade 3/4 non-hematologic toxicities occurring in three or more patients included febrile neutropenia, elevated alanine aminotransferase, hypokalemia, mucositis, and tumor lysis syndrome and occurred across all doses. Response and complete were observed at all dose levels with a $47 \%$ overall response rate and $27 \%$ complete response rate. Pharmacodynamic correlative studies demonstrated dose-dependent inhibition of PI3K/mTOR pathway phosphoproteins in all studied patients. Temsirolimus at doses up to $25 \mathrm{mg} / \mathrm{m}^{2}$ with cyclophosphamide and etoposide had an acceptable safety profile in children with relapsed/refractory ALL. Pharmacodynamic mTOR target inhibition was achieved and appeared to correlate with temsirolimus dose. Future testing of next-generation $\mathrm{PI} 3 \mathrm{~K} / \mathrm{mTOR}$ pathway inhibitors with chemotherapy may be warranted to increase response rates in children with relapsed/refractory ALL.
\end{abstract}




\section{Introduction}

Phosphatidylinositol 3-kinase (PI3K)/mammalian target of rapamycin (mTOR) signaling, a critical pathway in cell proliferation, metabolism, and apoptosis, is commonly dysregulated in acute lymphoblastic leukemia (ALL) and may confer chemotherapy resistance. ${ }^{1}$ While MTOR mutations are themselves uncommon in human cancer, other PI3K pathway gene mutations and expression changes that activate PI3K/mTOR signal transduction have been reported in many hematologic malignancies..$^{1-4}$ For example, loss of tumor suppressors that normally regulate PI3K signaling, such as PTEN (phosphatase and tensin homolog), can dysregulate normal cellular equilibrium and facilitate aberrant signaling activation. ${ }^{5}$ Constitutive PI3K/mTOR signaling activation in ALL may also result from increased surface expression of growth factor receptors on leukemia cells or from mutation of intracellular downstream effector genes. ${ }^{6}$ Preclinical studies of mTOR inhibitors in murine models of human ALL have shown potent in vivo inhibition of leukemia proliferation and prolonged animal survival in comparison to that of vehicle-treated controls. ${ }^{7-10}$

Despite aggressive retrieval strategies, the prognosis for children with relapsed/refractory ALL is poor.11-14 Molecularly-targeted agents, including mTOR inhibitors, have shown promise in treating some patients. ' Two pediatricspecific trials of mTOR inhibition in combination with chemotherapy have been performed to date. The Children's Oncology Group (COG) ADVL1114 phase I trial explored the safety and tolerability of combining three weekly doses of temsirolimus with UK ALLR3 reinduction therapy in children with second or greater relapse/refractory ALL (www.clinicaltrials.gov NCT01403415).15 The study required two de-escalations of temsirolimus down to 7.5 $\mathrm{mg} / \mathrm{m}^{2} /$ dose due to observed dose-limiting toxicity (DLT), and this regimen was determined to be too toxic for further assessment in a phase II clinical trial setting. DLT seen in this study specifically appeared to be exacerbated by the combinatorial toxicity of mTOR inhibitors with the known toxicities of steroids and asparaginase. Despite DLT, complete responses (CR) occurred in seven of 15 treated patients. Dose-dependent inhibition of PI3K/mTOR pathway signaling was also detected in most patients via correlative pharmacodynamic studies..$^{15}$ The Dana-Farber Cancer Institute Consortium (DFCl) 11-237 phase I trial (www.clinicaltrials.gov NCT01523977) combined daily oral everolimus with four-drug re-induction in pediatric patients with first relapse of ALL, ${ }^{16}$ which was tolerated well. Nineteen of 22 patients achieved CR, 12 with minimal residual disease (MRD) $<0.1 \%$.

The Therapeutic Advances in Childhood Leukemia and Lymphoma (TACL) Consortium conducted the 2014-001 phase I clinical trial to define the recommended phase 2 dose (RP2D) of temsirolimus in combination with cyclo- phosphamide and etoposide chemotherapy chosen as a non-steroid/non-asparaginase regimen to mitigate toxicity observed in the ADVL1114 study in children and adolescents with second or greater relapsed ALL. Exploratory study aims included preliminary assessment of treatment efficacy within the context of a phase I trial and pharmacodynamic measurement of $\mathrm{PI} 3 \mathrm{~K} / \mathrm{mTOR}$ signaling pathway inhibition.

\section{Methods}

\section{Eligibility}

Patients $\geq 1$ and $<21$ years of age with second or greater relapse or chemotherapy-refractory B-ALL or T-ALL were eligible for study participation. Relapsed leukemia was defined as $>25 \%$ blasts in bone marrow (M3) or $5-25 \%$ blasts in bone marrow (M2) with evidence of concurrent extramedullary disease. Refractory disease was allowed with no more than one prior failed salvage attempt following the current relapse or no more than two additional treatment cycles after initial induction failure in newly diagnosed patients. After temsirolimus dosing was shown to be tolerable at $10 \mathrm{mg} / \mathrm{m}^{2}$ (DL2), eligibility was amended to include T-ALL in first relapse, and marrow involvement for eligibility was changed to $\geq 5 \%$ blasts regardless of extramedullary leukemia involvement. The definition of refractory leukemia was also expanded to include patients with any relapse of $A L L$ with $M R D \geq 0.1 \%$ after a reinduction attempt and patients with newly-diagnosed ALL with persistent MRD $\geq 0.1 \%$ in bone marrow following high-risk ALL consolidation therapy.

Eligibility criteria included a Lansky/Karnofsky performance score $\geq 50$, recovery from acute toxic effects of prior therapy, and no active infections. Patients had to be $\geq 2$ weeks from prior cytotoxic therapy with the exception of maintenance-type ALL therapy for which there was no washout period. Intrathecal chemotherapy was allowed within 7 days of initiation of systemic therapy. Patients had to be $\geq 7$ or $\geq 14$ days from short-acting or long-acting growth factor therapy, respectively, $\geq 7$ days from biologic anti-neoplastic therapy, $\geq 30$ days from cellular immunotherapy, and $\geq 3$ half-lives from prior monoclonal antibody therapy. Patients also had to be $\geq 3$ months from prior hematopoietic stem cell transplantation (HSCT) and without evidence of graft-versus-host disease. Patients receiving corticosteroids must have been on a stable or decreasing dose for 7 days prior to enrollment. Hydroxyurea use was permitted until 24 hours prior to the first dose of study chemotherapy.

Other eligibility requirements included a normal age-adjusted serum creatinine or glomerular filtration rate $\geq 70$ $\mathrm{mL} / \mathrm{min} / 1.73 \mathrm{~m}^{2}$, normal cardiac function defined by shortening fraction $\geq 27 \%$ or ejection fraction $\geq 50 \%$, adequate 
pulmonary function with a baseline oxygen saturation $>94 \%$ on room air, and adequate liver function defined as total bilirubin $\leq 1.5$ times, $\gamma$-glutamyl transferase $\leq 2.5$ times, alanine amino transaminase and aspartate transaminase $\leq 3$ times the institutional upper limits of normal for age. Fasting serum triglyceride and cholesterol were required to be $\leq 300 \mathrm{mg} / \mathrm{dL}$, and a fasting glucose had to be within normal limits for age.

This phase I study was registered at www.clinicaltrials.gov (NCT01614197) and approved by the local institutional review boards at all participating centers. Written informed consent (and assent as appropriate) was obtained for treatment and for optional correlative biology studies from patients $\geq 18$ years or parents/legal guardians of children aged $<18$ years according to institutional policies and in accordance with the Declaration of Helsinki.

\section{Drug administration and study design}

The primary objectives of the study were: (i) to determine the maximum tolerated dose or highest tested dose of temsirolimus administered in combination with cyclophosphamide and etoposide in pediatric and young adult patients with relapsed/refractory ALL; and (ii) to define the DLT and describe other serious toxicities of temsirolimus when combined with cyclophosphamide and etoposide. The secondary objectives were: (i) to determine the CR rate, (ii) to measure MRD levels by flow cytometry after one cycle of therapy, and (iii) to evaluate single-cell pharmacodynamic PI3K/mTOR pathway inhibition in patients' lymphoblasts during administration of temsirolimus and chemotherapy.

A $3+3$ patient cohort escalation design was used. ${ }^{17}$ Temsirolimus was supplied by Pfizer, Inc. Commercially-available cyclophosphamide $\left(440 \mathrm{mg} / \mathrm{m}^{2}\right)$ and etoposide $\left(100 \mathrm{mg} / \mathrm{m}^{2}\right)$ were administered intravenously once daily on days 1-5. Temsirolimus was administered intravenously on day 1 (prior to chemotherapy) and as monotherapy on day 8 (Online Supplementary Figure S1). The starting dose of temsirolimus at dose level 1 (DL1) was $7.5 \mathrm{mg} / \mathrm{m}^{2} /$ dose based upon the prior combination trial toxicity ${ }^{15}$ and a lower dose than the Food and Drug Administration (FDA)-approved dose of $25 \mathrm{mg}$ weekly for adults with renal cell carcinoma. Dose escalation to $10 \mathrm{mg} / \mathrm{m}^{2} /$ dose (DL2), $15 \mathrm{mg} / \mathrm{m}^{2} /$ dose (DL3, equivalent to FDA-approved adult dosing of $25 \mathrm{mg}$ weekly), and $25 \mathrm{mg} / \mathrm{m}^{2} /$ dose (DL4) was planned. Temsirolimus dosing was capped at a maximal body surface area of $2 \mathrm{~m}^{2}$. No intra-patient dose escalation of temsirolimus was permitted. Intrathecal methotrexate was administered once via lumbar puncture on day -6 to 1 for all patients. If cerebrospinal fluid involvement was present (CNS2 or CNS3), intrathecal triple chemotherapy (cytarabine, hydrocortisone, methotrexate) was administered weekly until achievement of negative cerebrospinal fluid (CNS1). Enrolled patients were not permitted to receive non-protocol anti-cancer therapy, including tyrosine kinase inhibitors, while on study. Each chemotherapy cycle was 29 days long. If the patient had no evidence of progressive disease (defined as an increase of $>25 \%$ in the absolute number of bone marrow blasts or development of new extramedullary disease) he or she could receive a second cycle if recovered from all relevant toxicities. Therapy-associated toxicities were monitored in all administered treatment cycles but were only evaluated for DLT in cycle 1.

Toxicities were graded according to the Common Terminology Criteria for Adverse Events version 4.03 (http://ctep.cancer.gov). Hematologic DLT for patients with ALL was defined as bone marrow aplasia at day 42 or beyond not attributable to leukemic involvement. Nonhematologic DLT were defined as treatment-related grade $\geq 3$ adverse events at least possibly attributable to temsirolimus with exceptions for specific toxicities if they returned to grade $\leq 2$ by day 36 of protocol therapy. Any toxicity resulting in temsirolimus dose omission or those attributable to temsirolimus that did not resolve to grade $\leq 2$ by day 36 were considered dose-limiting.

Guidelines from the International Consensus Conference on Toxicity were utilized to identify expected toxicities of the multi-agent chemotherapy backbone and to help to define DLT of combination therapy. ${ }^{18}$ Grade 3 and 4 laboratory abnormalities included in this category were electrolyte abnormalities, elevated liver function tests, hypoalbuminemia, hypofibrinogenemia, fasting hyperglycemia, hypercholesterolemia, and hypertriglyceridemia. Toxicities common in children with relapsed ALL were excepted from DLT criteria, including grade 3 constitutional toxicities (fatigue, malaise, dehydration, weight loss) or gastrointestinal toxicities (nausea, vomiting, anorexia, diarrhea, mucositis) and grade $3 / 4$ fever, infection, and febrile neutropenia regardless of need for hospitalization. ${ }^{18}$

Disease evaluations were obtained at baseline and at the end of each cycle of therapy. CR was defined as bone marrow morphology with $<5 \%$ blasts (M1), no evidence of extramedullary disease, and recovery of peripheral blood counts (absolute neutrophil count $\geq 500 / \mu \mathrm{L}$ and a platelet count $\geq 50,000 / \mu \mathrm{L}$ independent of transfusion). A CR with incomplete hematologic recovery (CRi) was achievement of an M1 marrow and absence of extramedullary disease without normalization of absolute neutrophil count and/or platelet count. Partial response was defined as clearance of peripheral blasts with $5-25 \%$ residual blasts in bone marrow (M2) or an M1 bone marrow without complete eradication of extramedullary disease. Patients who failed to qualify as having a CR, CRi, or partial response were defined as having stable disease or progressive disease. Flow cytometric MRD assessment of bone marrow specimens with morphological CR/CRi at end-cycle 1 was performed at the University of Washington. MRD $<0.01 \%$ was considered negative. ${ }^{19,20}$ 


\section{Pharmacodynamic analyses}

Baseline and post-treatment peripheral blood and bone marrow specimens were obtained from consenting patients for assessment of in vitro and ex vivo inhibition of $\mathrm{PI} 3 \mathrm{~K} / \mathrm{mTOR}$ pathway phosphoproteins within ALL cells via single-cell phosphoflow cytometry assays as described elsewhere. ${ }^{15,21}$ Peripheral blood samples were obtained at three time points: immediately prior to temsirolimus therapy (day 0), at day 3-5 of therapy after the first dose of temsirolimus, and at day 29 at the end of re-induction therapy (Online Supplementary Figure S1). Specimens at each timepoint were processed immediately upon receipt and stored for subsequent batched phosphoflow cytometry analysis of all samples from each temsirolimus dose level.

\section{Results}

\section{Patients' characteristics}

Sixteen patients aged 2-19 years (median 10 years) were enrolled between June 2015 and September 2019 (Table 1). One patient chose not to initiate protocol therapy after signing consent and was thus not evaluable. Ten evaluable patients had relapsed/refractory B-ALL, and five had relapsed/refractory T-ALL (Table 2). One patient was in first relapse, eight were in second relapse, and six were in third or subsequent relapse, of whom two patients were refractory to prior therapy. Patients had previously undergone a median of three salvage chemotherapy regimens prior to study entry (range, 2-7). Eight of 15 patients had relapsed after HSCT, and four patients had received prior CD19-targeted chimeric antigen receptor T-cell immunotherapy (CD19, targeted CAR T cells). All patients had $>25 \%$ bone marrow involvement (median 60\% bone marrow blasts) and were CNS1 $(n=14)$ or CNS2 $(n=2)$ at enrollment. Six patients had unfavorable ALL-associated genetic alterations, including the $B C R-A B L 1$ rearrangement or Philadelphia chromosome-like kinase fusions. All evaluable patients completed one cycle of therapy, and two patients received a second cycle on study.

\section{Toxicity assessment}

No patient treated at DL1 or DL2 experienced DLT. A patient at DL3 developed dose-limiting pneumonitis and pleural and pericardial effusions without an infectious organism being identified. Three additional patients were enrolled at DL3, none of whom experienced DLT. Based in part upon safety data in the first three dose level cohorts and real-time pharmacodynamic studies described below, temsirolimus dosing was subsequently explored at DL4 (above the FDA-approved adult dosing) in three patients for biology, toxicity, and response assessment with no DLT observed.
Table 3 delineates non-dose-limiting grade $3 / 4$ nonhematologic toxicities that were at least possibly attributed to temsirolimus and occurred in $>10 \%$ of patients during cycle 1 of therapy. The most common non-hematologic toxicities were febrile neutropenia (67\%) and infection/sepsis (40\%). The majority of blood infections were due to Gram-positive cocci. Two Gram-negative infections occurred; one patient had Pseudomonas aeruginosa bacteremia, urinary tract infection, and labial wound infection, and a second patient had Klebsiella pneumoniae bacteremia. Three patients had Clostridium difficile-associated enterocolitis. Four patients had viral infections caused by rhinovirus $(n=2)$, influenza $B(n=1)$, and enterovirus $(n=1)$. Electrolyte and metabolic abnormalities included hyperkalemia (40\%), hypophosphatemia (20\%), tumor lysis syndrome (20\%), and hyperglycemia (13\%). Gastrointestinal toxicities included mucositis (27\%), elevated alanine transaminase (27\%), abdominal pain (20\%), and elevated $\gamma$-glutamyl transferase (13\%). Hypertriglyceridemia and hypercholesterolemia were not observed.

\section{Responses}

Clinical responses were observed in patients treated at all dose levels of temsirolimus (Table 2). The overall response rate (CR + Cri + partial response) was $47 \%$, occurring in three of five patients with T-ALL and four of ten patients with B-ALL. Three of the four patients with CR or CRi also had MRD $<0.01 \%$, and two of these patients underwent subsequent allogeneic HSCT.

Table 1. Summary of characteristics of patients enrolled on TACL 2014-001.

\begin{tabular}{|l|c|}
\hline Characteristic & Total N=15 \\
\hline $\begin{array}{l}\text { Age at enrollment, years } \\
\text { Median } \\
\text { Range }\end{array}$ & 10 \\
\hline Sex, N & $2-19$ \\
Male & \\
Female & 9 \\
\hline Race, N (\%) & 6 \\
White & \\
Asian/Pacific Islander & $8(53 \%)$ \\
Black or African American & $2(14 \%)$ \\
Other/Unknown & $1(7 \%)$ \\
\hline Ethnicity, N (\%) & $4(26 \%)$ \\
\hline Non-Hispanic & \\
Hispanic & $7(47 \%)$ \\
Unknown & $7(47 \%)$ \\
\hline Prior therapy regimens, N (\%) & $1(6 \%)$ \\
Median & \\
Range & 3 \\
Prior transplant & $2-7$ \\
Prior CAR T-cell & $8 / 15(53 \%)$ \\
\hline
\end{tabular}

CAR: chimeric antigen receptor. 
Table 2. Disease status and clinical responses of patients treated on TACL 2014-001.

\begin{tabular}{|c|c|c|c|c|c|}
\hline Dose Level & USI & Diagnosis & Relapse ${ }^{\#}$ & ALL-associated genetic alterations & End-Cycle 1 response \\
\hline \multirow{3}{*}{$\mathrm{DL} 1$} & 1 & B-ALL & 2 & Complex cytogenetics & PR \\
\hline & 2 & B-ALL & $>4$ & ETV6-RUNX1 & PD \\
\hline & 3 & T-ALL & 2 & na & CRi, MRD 2.4\% \\
\hline \multirow{3}{*}{ DL2 } & 5 & B-ALL & 2 & KMT2A rearrangement & $\mathrm{CR}, \mathrm{MRD}<0.01$ \\
\hline & 6 & T-ALL & 2 & na & SD \\
\hline & 7 & B-ALL & 3 & $B C R-A B L 1$ & PR \\
\hline \multirow{6}{*}{ DL3 } & 8 & B-ALL & 2 & na & PD \\
\hline & $9^{*}$ & B-ALL & 3 ref & EBF1-PDGFRB & SD \\
\hline & 10 & T-ALL & 1 & na & PR \\
\hline & 11 & B-ALL & 3 ref & ETV6-RUNX1 & PD \\
\hline & 12 & B-ALL & 2 & P2RY8-CRLF2 & $\mathrm{CR}, \mathrm{MRD}<0.01$ \\
\hline & 13 & B-ALL & 2 & $R C S D 1-A B L 2$ & PD \\
\hline \multirow{3}{*}{ DL4 } & 14 & T-ALL & $>4$ & na & SD \\
\hline & 15 & T-ALL & 2 & na & CRi, MRD <0.01 \\
\hline & 16 & B-ALL & $>3$ & Ph-like ABL class & PD \\
\hline
\end{tabular}

*Patient with dose limiting toxicity, ALL: acute lymphoblastic leukemia; CR: complete response; CRi: complete response with incomplete platelet recovery; DL: dose level; MRD: minimal residual disease; na: not available; PD: progressive disease; ref: refractory; SD: stable disease; USI: unique specimen identifier.

\section{Pharmacodynamic studies}

Peripheral blood samples from consenting patients with

Table 3. Non-dose-limiting non-hematologic toxicities related to protocol therapy and observed in $>10 \%$ of evaluable patients $(n=15)$.

\begin{tabular}{|l|c|c|c|c|}
\hline Toxicity & Grade 1/2 & Grade 3 & Grade 4 & \% Gr 3/4 \\
\hline $\begin{array}{l}\text { Febrile } \\
\text { neutropenia }\end{array}$ & 0 & 9 & 1 & $67 \%$ \\
\hline Infection/sepsis & 1 & 5 & 1 & $40 \%$ \\
\hline Hyperkalemia & 4 & 2 & 4 & $40 \%$ \\
\hline Mucositis & 6 & 4 & 0 & $27 \%$ \\
\hline ALT increase & 5 & 3 & 1 & $27 \%$ \\
\hline $\begin{array}{l}\text { Hypo- } \\
\text { phosphatemia }\end{array}$ & 2 & 2 & 1 & $20 \%$ \\
\hline $\begin{array}{l}\text { Tumor lysis } \\
\text { syndrome }\end{array}$ & 0 & 3 & 0 & $20 \%$ \\
\hline Abdominal pain & 4 & 3 & 0 & $20 \%$ \\
\hline$\gamma$ GT increased & 4 & 2 & 0 & $20 \%$ \\
\hline Hyperglycemia & 5 & 2 & 0 & $13 \%$ \\
\hline Hypoxia & 0 & 2 & 0 & $13 \%$ \\
\hline
\end{tabular}

*Three patients had multiple infectious incidents; one with the pseudomonas at three different sites, and two with grade 3 Clostridium difficile enterocolitis. Maximum infection grade included in table. Gr: grade; ALT: alanine transaminase; $\gamma \mathrm{GT}$ : gamma glutamyltransferase.
B-ALL $(n=7)$ and T-ALL $(n=2)$ were obtained from patients treated at DL1 $(n=1), \operatorname{DL} 2(n=1), \operatorname{DL} 3(n=6)$, and DL4 $(n=1)$ and analyzed by leukemia cell-specific phosphoflow cytometry. We observed basal activation of PI3K/mTOR pathway phosphoproteins in all tested samples at the day 0 pre-treatment time point (Figure 1). Although limited by small numbers, a trend towards dose-dependent in vivo inhibition of phosphosignaling was detected in studied specimens at 3-5 days after the first dose of temsirolimus (Figure 2; summary data in Online Supplementary Figure S2). In vitro incubation of pre-treatment and day 3-5 blood samples with the mTOR inhibitor rapamycin performed to define maximal achievable PI3K pathway signaling inhibition for each patient's leukemia cells further supported clinical temsirolimus dose escalation with greatest inhibition detected in vivo for a patient treated at DL4 (Figure 2). Correlative blood specimens were not submitted for three of the four patients who achieved CR/CRi, so it was unfortunately not possible to assess potential correlation of pharmacodynamic signaling inhibition with clinical responses.

\section{Recommended phase 2 dose determination}

Based upon safety data with DLT assessment, pharmacodynamic assay results, and evaluation of dosing higher than that approved by the FDA in the DL4 cohort, temsirolimus $15 \mathrm{mg} / \mathrm{m}^{2}$ (DL3) in combination with cyclophosphamide/etoposide was selected as the RP2D for future clinical trial investigation. 


\section{Discussion}

Therapy for children and adolescents with multiply relapsed ALL is hampered by low remission rates and significant risk of morbidity and mortality with intensive salvage therapy. ${ }^{13-15,}$,22-24 Many promising molecularly targeted agents have been approved for adults with a variety of cancers, but these likely must be combined with multiagent cytotoxic chemotherapy regimens to improve longterm survival appreciably in children with ALL. The TACL 2014-001 phase I trial was conducted to evaluate the safety and tolerability of the MTOR inhibitor temsirolimus in combination with the commonly used cyclophosphamide and etoposide salvage regimen for relapsed/refractory ALL.

In this study, we observed acceptable safety and tolerability of temsirolimus on this backbone regimen, as well as clinical responses in nearly half of treated patients. Due to the observed prior toxicity of temsirolimus with other combination chemotherapy regimens in early-phase pediatric oncology trials, we chose a priori to decrease the number of weekly doses of temsirolimus from three to two when designing this protocol. An identical overall response rate $(47 \%)$ in this trial using two doses of temsirolimus on the cyclophosphamide/etoposide backbone was reported among patients on the COG ADVL1114 trial initially using three (and then two) doses of temsirolimus combined with the more toxic UK ALLR3 reinduction chemotherapy platform. ${ }^{15}$ We demonstrate that two weekly temsirolimus doses may be sufficiently effective on a less toxic chemotherapy backbone in a similar re- lapsed/refractory pediatric population. Although no DLT were observed in the three patients treated at DL4 (25 $\mathrm{mg} / \mathrm{m}^{2} /$ dose; the highest tested dose), the RP2D of temsirolimus in combination with cyclophosphamide and etoposide from this trial is $15 \mathrm{mg} / \mathrm{m}^{2} /$ dose (DL3), which is consistent with the defined RP2D in adult patients. Given the observed intolerability of lower temsirolimus dosing with more intensive chemotherapy in our prior ADVL1114 phase I clinical trial,,$^{15}$ temsirolimus $15 \mathrm{mg} / \mathrm{m}^{2} /$ dose for two doses represents an appropriately cautious recommendation for incorporation into ALL salvage regimens.

Combining temsirolimus with cyclophosphamide and etoposide avoided many severe combinatorial toxicities, such as hyperglycemia, hypertriglyceridemia, mucositis, and poor wound healing, reported in earlier studies combining mTOR inhibition with other intensive chemotherapy regimens. ${ }^{15,25,26} \mathrm{~A}$ pediatric phase I trial of temsirolimus combined with irinotecan and temozolomide in children with relapsed/refractory solid tumors similarly required modification to exclude patients on concomitant steroids due to dose-limiting hyperlipidemia. ${ }^{27}$ However, combination of the oral mTOR inhibitor everolimus with a four-drug re-induction in children with a first relapse of ALL in the DFCI trial 11-237 appeared well-tolerated with less metabolic toxicity, ${ }^{16}$ suggesting that the number of relapses and therapy lines may also influence toxicity. Several recent relapsed pediatric ALL trials and reviews have reported 4$5 \%$ mortality rates and $45-92 \%$ rates of grade $3-4$ infections during re-induction therapy. ${ }^{22,23}$ On our trial, expected rates of febrile neutropenia (67\%) and grade $3 / 4$ infection $(40 \%)$, with viral and bacterial etiologies, were observed.

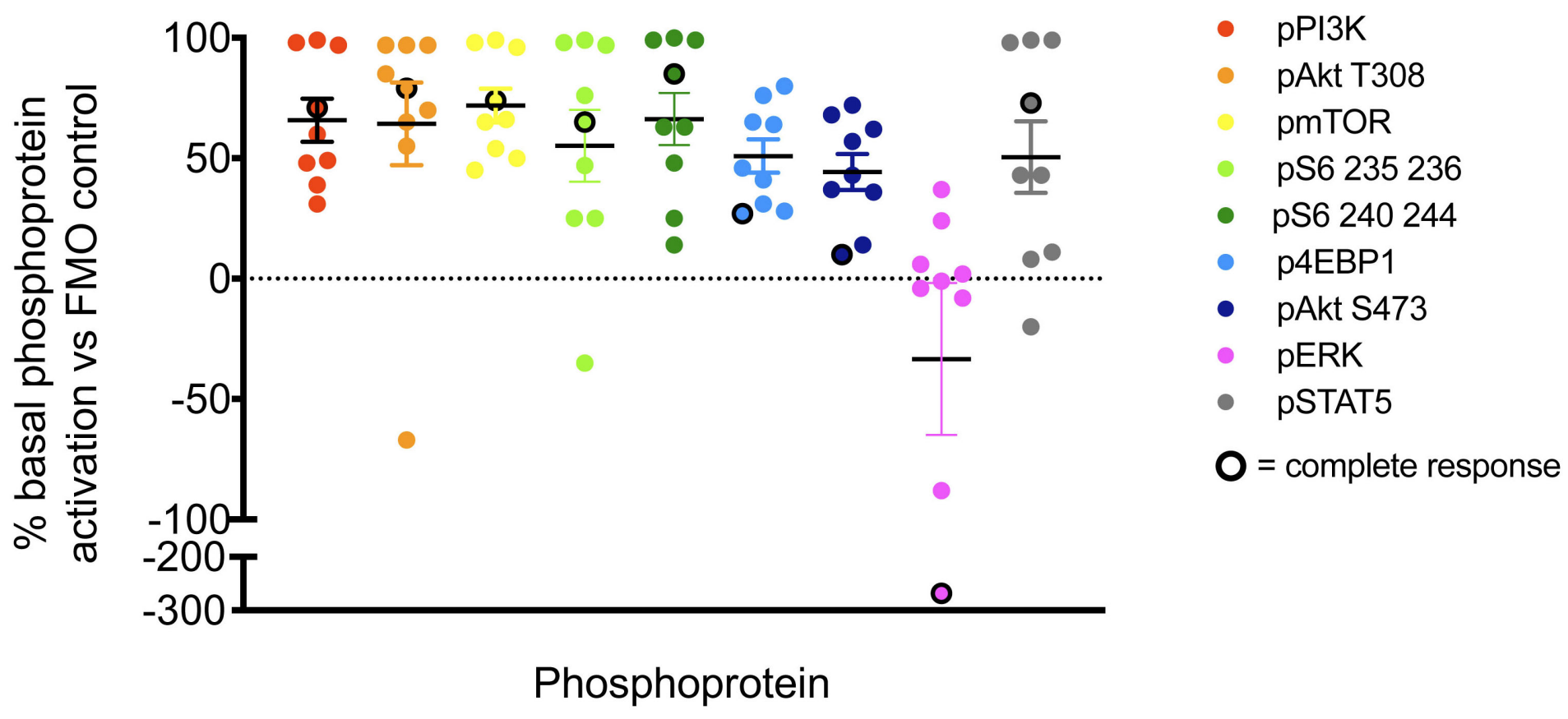

Figure 1. Constitutive activation of PI3K pathway signaling in children with relapsed/refractory acute lymphoblastic leukemia enrolled on TACL2014-001. Pre-treatment (basal, day 0) peripheral blood samples were obtained from study patients with acute lymphoblastic leukemia (ALL) for single-cell phosphoflow cytometry analysis of the PI3K pathway and other phosphoproteins as previously described..$^{15}$ Pre-treatment blood specimens from most patients show basal activation of multiple PI3K/mTOR pathway phosphoproteins in gated human leukemia cells (CD45 $/$ CD $19^{+} B-A L L$ or CD $45^{+} / C D 3^{+}$for T-ALL) when compared to fluorescenceminus-one (FMO)-stained control cells. Solid symbols represent patients with partial response, stable disease, or progressive disease after cycle 1. Black-ringed symbols represent patients with complete response. 
Clinical responses were observed in patients treated at every dose level with an overall response rate of $47 \%$ and a CR/CRi of $27 \%$ and did not appear to be temsirolimus dose-related, further supporting the selected RP2D. Achievement of MRD-negative remission with study therapy also facilitated subsequent allogeneic HSCT in two patients. Although MRD-negative $\mathrm{CR}$ are the "gold standard" to enable consolidative HSCT, partial responses or even stable disease in patients with B-ALL can serve as meaningful clinical outcomes that enable disease stability as a bridge to CAR T-cell immunotherapy during the manufacturing process. Although limited by small numbers, the CR rate of patients with T-ALL ( $40 \%$, one of whom achieved morphological remission but remained MRD positive) in this study was encouraging given the historic difficulty in successful salvage of children with relapsed T-ALL. ${ }^{28}$ Not unexpectedly, higher rates of clinical response occurred in patients in second relapse (6 of 9) compared to those in third or subsequent relapse (1 of 6), which is consistent with prior literature. ${ }^{14,28}$ Four of eight patients who had relapsed after allogeneic HSCT responded to the temsirolimus, cyclophosphamide, and etoposide study regimen, whereas the four patients with B-ALL who had relapsed after CD19-targeted CAR T cells had progressive disease on the current trial.

The protocol was amended to incorporate more modern pediatric definitions of relapse including morphological disease of $\geq 5 \%$ or COG-certified flow cytometry MRD of $\geq 0.1 \%$ in accordance with a recent international consensus study by the Ponte-di-Legno Consortium. ${ }^{29}$ Despite the study amendment, all patients who enrolled on TACL2014$001 \mathrm{had} \geq 25 \%$ bone marrow involvement. Future leukemia trials will need to assess whether patients with lower disease burden at time of enrollment using more modern definitions of relapse will have differential response rates and/or less trial toxicity when treated with lower disease burden.

In summary, we report that the mTOR inhibitor temsirolimus can be safely administered as two weekly doses in combination with 5 days of cyclophosphamide and eto-
DL1 $7.5 \mathrm{mg} / \mathrm{m}^{2}$ temsirolimus

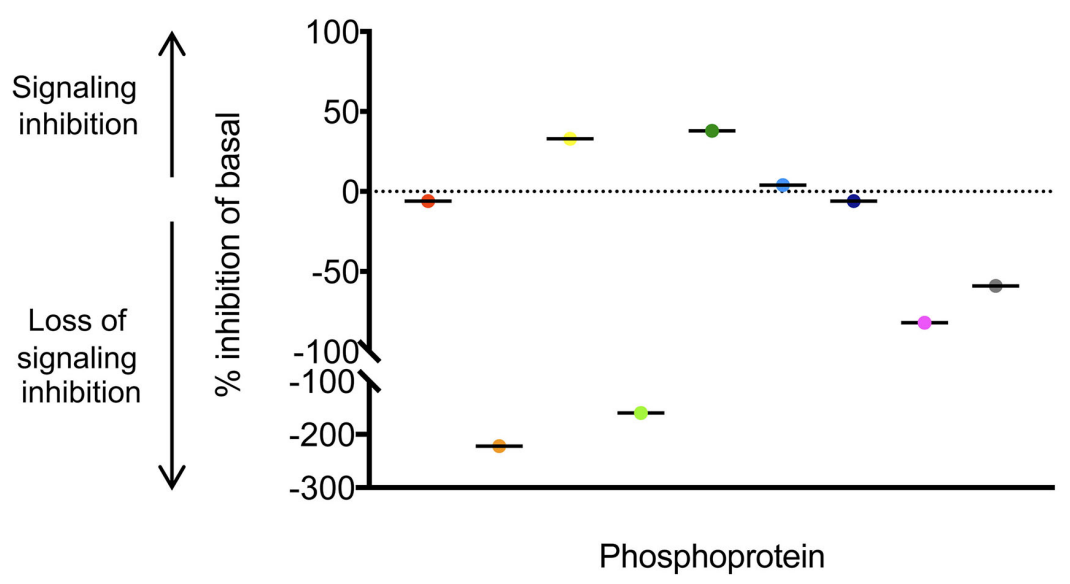

DL3 $15 \mathrm{mg} / \mathrm{m}^{2}$ temsirolimus

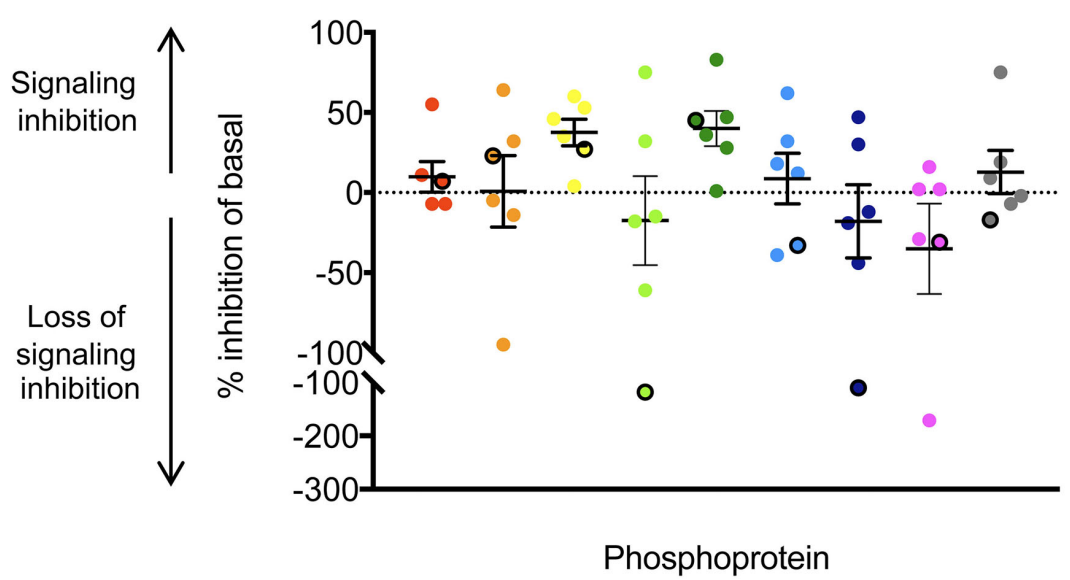

$\mathrm{DL} 210 \mathrm{mg} / \mathrm{m}^{2}$ temsirolimus

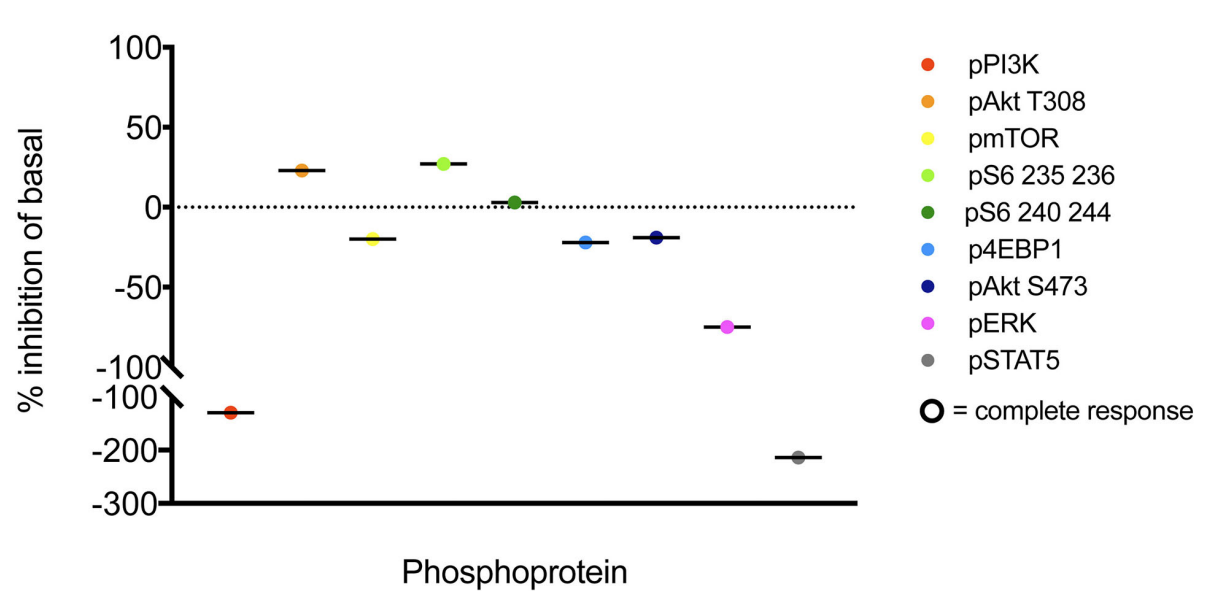

DL4 $25 \mathrm{mg} / \mathrm{m}^{2}$ temsirolimus

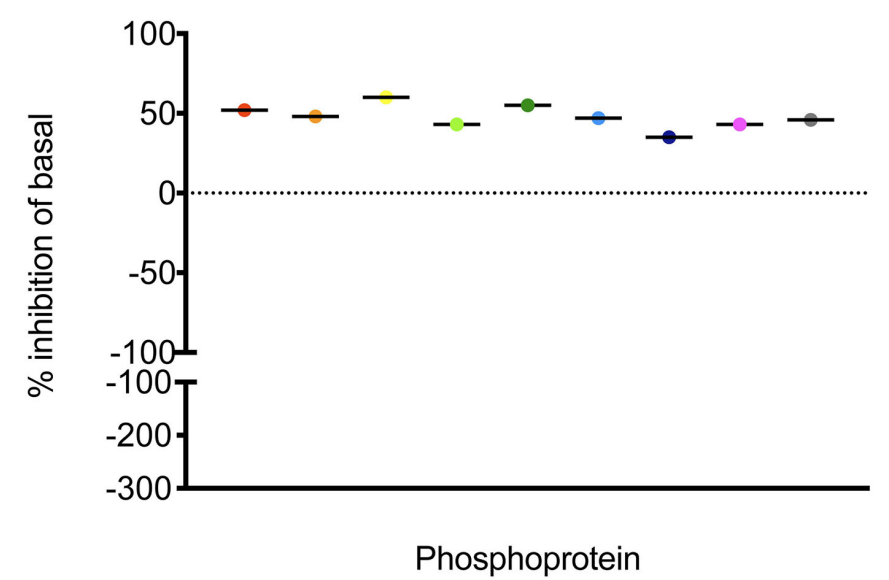

Figure 2. Abrogation of constitutively-activated PI3K/mTOR pathway signaling with temsirolimus therapy. Pre-treatment (basal, day 0 ) and post-treatment (day 3-5) levels of PI3K/mTOR pathway phosphoproteins were measured as median fluorescence intensity (MFI) by single-cell phosphoflow cytometry in gated B-acute lymphoblastic leukemia (ALL) or T-ALL cells in peripheral blood specimens from TACL2014-001 patients. Phosphoprotein inhibition in peripheral blood ALL cells at day 3-5 of therapy after the first dose of temsirolimus in comparison to basal phosphoprotein levels is shown for each patient treated at the designated dose levels (DL1, DL2, DL3, DL4). MFI data were normalized intra-patient to pre-treatment levels of each phosphoprotein. Central horizontal solid lines depict mean phosphoprotein inhibition for inter-patient comparison. Dotted line set at $y=0$ indicates no change in phosphoprotein from baseline. Solid symbols represent patients with partial response, stable disease, or progressive disease after cycle 1. Black-ringed symbols represent patients with complete response. Summary pharmacodynamic data of all dose levels are shown in Online Supplementary Figure S2. 
poside, a commonly used chemotherapy backbone regimen for patients with relapsed/refractory ALL, with a temsirolimus RP2D of $15 \mathrm{mg} / \mathrm{m}^{2} /$ dose. Our small correlative pharmacodynamic dataset also identified elevated basal PI3K/mTOR phosphoprotein levels in all studied patients with seemingly dose-dependent in vivo signaling inhibition after temsirolimus being detected. The contribution of temsirolimus to the responses observed in our study seems biologically plausible given known mTOR pathway activation in children with relapsed ALL and merits more detailed exploration in future larger studies. ${ }^{1,30}$ Future trials may also explore targeting of more proximal or multiple proteins in the $\mathrm{PI} 3 \mathrm{~K} / \mathrm{mTOR}$ pathway or combination of mTOR inhibitors with other signaling pathway inhibitors, such as JAK- or ABL-targeted inhibitors ${ }^{31-33}$ with goals of improving deep remission rates and possibly less dependence on conventional cytotoxic chemotherapy to achieve cure.

\section{Disclosures}

SKT receives research funding from Incyte Corporation, Gilead Sciences, and MacroGenics, has consulted for Kura Oncology, and is a scientific advisory board member at Aleta Biotherapeutics. LBS receives research funding from Servier and has received consulting fees from Takeda, Servier, and Syndax. JAW receives research funding from Novartis and Daichii for unrelated studies and consulting fees from Amgen. KRS receives consulting fees from Juno/BMS as a data safety monitoring board member for unrelated clinical trials. He has also received consulting fees from Jazz, Janssen, Incyte, and Novartis for unrelated studies. $E O$ is a scientific advisory board member for Jazz Pharmaceuticals and receives research and consulting funding from Servier Pharmaceuticals unrelated to this work. CMB receives research funding from Merck Sharp and Dohme for unrelated studies. TWL has received consulting funding from Novartis, Pfizer, Loxo Oncology/Eli Lilly, Bayer, and Cellectis and research funding from Novartis, Pfizer, and Bayer. TMC receives consulting fees from Kura Oncology. He also acknowledges employment of spouse by Juno/Celgene until August 2019 ASW receives research funding from
Kite Pharma and Institut de Recherches Internationales Servier. SRR receives research and consulting funding from Pfizer, Inc for this and unrelated studies. She also acknowledges employment of spouse by OptiNose, Inc. The remaining authors declare no financial conflicts of interest.

\section{Contributions}

$S K T, L B S, J A W$, and SRR designed and performed the research, analyzed data, and wrote the clinical protocol and manuscript. JPL conducted experiments and analyzed data. RS, JM, and YC analyzed data and performed statistical analyses. EO, ESS, KRS, RJH, PG, CMB, TMC, TWL, MLS, and ASW enrolled patients on study, contributed data, and edited the manuscript. All authors approved the final version of the manuscript. .

\section{Acknowledgements}

We gratefully acknowledge the patients and families who participated in the clinical trial. We acknowledge the TACL Consortium's scientific contribution to and participation in this study, including participating member institutions, investigators, research teams, and the TACL Operations Center. We thank Mr. John Chukinas and Dr. David Hottman Allen at the Children's Hospital of Philadelphia for assistance with correlative biology studies.

\section{Funding}

Research reported in this publication was supported by the Pfizer 2B clinical trial program, NCl award P30CA014089, the Higgins Family Foundation, and the Leukemia \& Lymphoma society. SKT was supported by $\mathrm{NIH/NCI}$ K08CA184418, R21HD081319, U01CA232486, U01CA243072, Department of Defense Translational Team Science Award CA180683P1, the Rally Foundation for Childhood Cancer Research, and the $V$ Foundation for Cancer Research.

\section{Data-sharing statement}

Qualified researchers can request reasonable access to de-identified patient-level clinical data that support the manuscript and the clinical trial protocol from the corresponding author (SRR).

\section{References}

1. Tasian SK, Teachey DT, Rheingold SR. Targeting the PI3K/mTOR pathway in pediatric hematologic malignancies. Front Oncol. 2014;4:108.

2. Bjornsti MA, Houghton PJ. The TOR pathway: a target for cancer therapy. Nat Rev Cancer. 2004;4(5):335-348.

3. Smolewski P. Investigating mammalian target of rapamycin inhibitors for their anticancer properties. Expert Opin Investig Drugs. 2006;15(10):1201-1227.

4. Grabiner BC, Nardi V, Birsoy K, et al. A diverse array of cancerassociated MTOR mutations are hyperactivating and can predict rapamycin sensitivity. Cancer Discov. 2014;4(5):554-563.

5. Gutierrez A, Sanda T, Grebliunaite R, et al. High frequency of PTEN, PI3K, and AKT abnormalities in T-cell acute lymphoblastic leukemia. Blood. 2009;114(3):647-650.

6. Chapuis N, Tamburini J, Cornillet-Lefebvre P, et al. Autocrine IGF-1/IGF-1R signaling is responsible for constitutive PI3K/Akt activation in acute myeloid leukemia: therapeutic value of neutralizing anti-IGF-1R antibody. Haematologica.

2010;95(3):415-423.

7. Teachey DT, Obzut DA, Cooperman J, et al. The mTOR inhibitor 
$\mathrm{CCl}-779$ induces apoptosis and inhibits growth in preclinical models of primary adult human ALL. Blood.

2006;107(3):1149-1155.

8. Houghton PJ, Morton CL, Kolb EA, et al. Initial testing (stage 1) of the MTOR inhibitor rapamycin by the pediatric preclinical testing program. Pediatr Blood Cancer. 2008;50(4):799-805.

9. Teachey DT, Sheen C, Hall J, et al. mTOR inhibitors are synergistic with methotrexate: an effective combination to treat acute lymphoblastic leukemia. Blood. 2008;112(5):2020-2023.

10. Crazzolara R, Cisterne A, Thien M, et al. Potentiating effects of RAD001 (everolimus) on vincristine therapy in childhood acute lymphoblastic leukemia. Blood. 2009;113(14):3297-3306.

11. Chessells JM. Relapsed lymphoblastic leukaemia in children: a continuing challenge. Br J Haematol. 1998;102(2):423-438.

12. Gaynon PS, Qu RP, Chappell RJ, et al. Survival after relapse in childhood acute lymphoblastic leukemia: impact of site and time to first relapse--the Children's Cancer Group experience. Cancer. 1998;82(7):1387-1395.

13. Reismuller B, Peters C, Dworzak MN, et al. Outcome of children and adolescents with a second or third relapse of acute lymphoblastic leukemia (ALL): a population-based analysis of the Austrian ALL-BFM (Berlin-Frankfurt-Munster) study group. J Pediatr Hematol Oncol. 2013;35(5):e200-204.

14. Sun W, Malvar J, Sposto R, et al. Outcome of children with multiply relapsed B-cell acute lymphoblastic leukemia: a Therapeutic Advances in Childhood Leukemia \& Lymphoma study. Leukemia. 2018;32(11):2316-2325.

15. Rheingold SR, Tasian SK, Whitlock JA, et al. A phase 1 trial of temsirolimus and intensive re-induction chemotherapy for 2 nd or greater relapse of acute lymphoblastic leukaemia: a Children's Oncology Group study (ADVL1114). Br J Haematol. 2017;177(3):467-474.

16. Place AE, Pikman Y, Stevenson KE, et al. Phase I trial of the mTOR inhibitor everolimus in combination with multi-agent chemotherapy in relapsed childhood acute lymphoblastic leukemia. Pediatr Blood Cancer. 2018;65(7):e27062.

17. Le Tourneau C, Lee JJ, Siu LL. Dose escalation methods in phase I cancer clinical trials. J Natl Cancer Inst. 2009;101(10):708-720.

18. Horton TM, Sposto R, Brown P, et al. Toxicity assessment of molecularly targeted drugs incorporated into multiagent chemotherapy regimens for pediatric acute lymphocytic leukemia (ALL): review from an international consensus conference. Pediatr Blood Cancer. 2010;54(7):872-878.

19. Borowitz MJ, Devidas M, Hunger SP, et al. Clinical significance of minimal residual disease in childhood acute lymphoblastic leukemia and its relationship to other prognostic factors: a Children's Oncology Group study. Blood. 2008;111(12):5477-5485.

20. Borowitz MJ, Wood BL, Devidas M, et al. Prognostic significance of minimal residual disease in high risk B-ALL: a report from Children's Oncology Group study AALL0232. Blood.
2015;126(8):964-971.

21. Loh ML, Tasian SK, Rabin KR, et al. A phase 1 dosing study of ruxolitinib in children with relapsed or refractory solid tumors, leukemias, or myeloproliferative neoplasms: A Children's Oncology Group phase 1 consortium study (ADVL1011). Pediatr Blood Cancer. 2016;62(10):1717-1724.

22. Raetz EA, Borowitz MJ, Devidas M, et al. Reinduction platform for children with first marrow relapse of acute lymphoblastic leukemia: a Children's Oncology Group Study [corrected]. J Clin Oncol. 2008;26(24):3971-3978.

23. Messinger YH, Gaynon PS, Sposto R, et al. Bortezomib with chemotherapy is highly active in advanced B-precursor acute lymphoblastic leukemia: Therapeutic Advances in Childhood Leukemia \& Lymphoma (TACL) study. Blood. 2012;120(2):285-290.

24. Parker C, Waters R, Leighton C, et al. Effect of mitoxantrone on outcome of children with first relapse of acute lymphoblastic leukaemia (ALL R3): an open-label randomised trial. Lancet. 2010;376(9757):2009-2017.

25. Hudes G, Carducci M, Tomczak P, et al. Temsirolimus, interferon alfa, or both for advanced renal-cell carcinoma. N Engl J Med. 2007;356(22):2271-2281.

26. Spunt SL, Grupp SA, Vik TA, et al. Phase I study of temsirolimus in pediatric patients with recurrent/refractory solid tumors. $J$ Clin Oncol. 2011;29(21):2933-2940.

27. Bagatell R, Norris R, Ingle AM, et al. Phase 1 trial of temsirolimus in combination with irinotecan and temozolomide in children, adolescents and young adults with relapsed or refractory solid tumors: a Children's Oncology Group Study. Pediatr Blood Cancer. 2014;61(5):833-839.

28. Nguyen K, Devidas M, Cheng SC, et al. Factors influencing survival after relapse from acute lymphoblastic leukemia: a Children's Oncology Group study. Leukemia. 2008;22(12):2142-2150.

29. Buchmann S, Schrappe M, Baruchel A, et al. Remission, treatment failure, and relapse in pediatric ALL: an international consensus of the Ponte-di-Legno Consortium. Blood. 2022;139(12):1785-1793.

30. Fruman DA, Rommel C. PI3K and cancer: lessons, challenges and opportunities. Nat Rev Drug Discov. 2014;13(2):140-156.

31. Tasian SK, Doral MY, Borowitz MJ, et al. Aberrant STAT5 and $\mathrm{PI3K} / \mathrm{mTOR}$ pathway signaling occurs in human CRLF2rearranged B-precursor acute lymphoblastic leukemia. Blood. 2012;120(4):833-842.

32. Tasian SK, Teachey DT, Li Y, et al. Potent efficacy of combined $\mathrm{PI} 3 \mathrm{~K} / \mathrm{mTOR}$ and JAK or $\mathrm{ABL}$ inhibition in murine xenograft models of Ph-like acute lymphoblastic leukemia. Blood. 2017;129(2):177-187.

33. Hurtz C, Wertheim GB, Loftus JP, et al. Oncogene-independent BCR-like signaling adaptation confers drug resistance in Ph-like ALL. J Clin Invest. 2020;130(7):3637-3653. 